\title{
An in vitro cytologic assay for evaluation of the KIT signaling pathway in gastrointestinal stromal tumors
}

\author{
Brian S Chang, Tao Yang, Edmund S Cibas and Jonathan A Fletcher \\ Department of Pathology, Brigham and Women's Hospital and Harvard Medical School, Boston, MA, USA
}

\begin{abstract}
Specific inhibitors can be designed to inactivate the molecular pathways involved in tumor growth. A compelling example is the use of small molecule drugs, such as imatinib (Gleevec), which inhibit the KIT tyrosine kinase in gastrointestinal stromal tumors (GIST). Assays are needed to determine which inhibitor is most effective at silencing the KIT kinase in each GIST patient. The aim of this study was to develop a robust, cytology-based assay to measure tumor susceptibility to target-specific small molecule inhibitors. We created an immortal GIST cell line (GIST882) that was treated in vitro with several inhibitors of the KIT $\rightarrow$ AKT $\rightarrow$ mTOR $\rightarrow$ S6 signaling pathway. KIT was inhibited with imatinib, and mTOR with RAD001. Treatment response was assessed in cytologic preparations by immunocytochemical staining with antibodies to KIT, phospho-KIT, phospho-AKT, and phospho-S6. Optimization was performed to maximize staining in the absence of inhibitor, and minimize staining in the presence of inhibitor. GIST882 cells demonstrated strong, robust phospho-S6 expression in the absence of inhibitor. This expression was completely inhibited by treatment with upstream signaling pathway inhibitors (imatinib and RAD001). Other phospho-specific antibodies had weaker baseline reactivity in the absence of inhibitor. The accuracy of the immunocytochemical results on the cytologic preparations was validated by immunoblotting studies. Our study demonstrates the feasibility of cytologic methods to monitor labile biochemical responses in tumor cells during drug therapy. Such approaches will be enhanced by the development of additional activation state-specific antibodies, particularly those optimized for use in cytologic preparations.

Modern Pathology (2007) 20, 579-583. doi:10.1038/modpathol.3800779; published online 30 March 2007
\end{abstract}

Keywords: gastrointestinal stromal tumor; cytology; immunocytochemistry; KIT pathway; tyrosine kinase; imatinib

Targeted drug therapy is the holy grail of oncology. Through an understanding of the key molecular pathways involved in tumor growth, specific inhibitors can be designed to inactivate these pathways, thereby maximizing therapeutic response. One of the most compelling examples of successful targeted therapy for a metastatic solid tumor is that of imatinib (Gleevec) inhibition of the KIT tyrosine kinase in gastrointestinal stromal tumors (GIST). ${ }^{1}$ Dramatic clinical responses are seen routinely after initiation of imatinib therapy, but most patients eventually relapse as the tumors develop resistance to the drug. Sunitinib (Sutent) was also recently FDA-approved for this clinical indication, and

Correspondence: Dr ES Cibas, MD, Department of Pathology, Brigham and Women's Hospital, 75 Francis St., Boston, MA 02115, USA.

E-mail: ecibas@partners.org

Received 25 January 2007; revised and accepted 20 February 2007; published online 30 March 2007 additional KIT kinase inhibitors are currently undergoing clinical trials.

To extend the clinical response, it will likely be necessary to administer multiple agents capable of targeting different tumor growth signaling pathways. Using GIST as a model system, investigators have identified several other key kinases that are important in cell proliferation and survival. ${ }^{2}$ Once the inhibitors of these molecules have been validated in ongoing clinical trials, oncologists will have the flexibility to design unique regimens in response to the specific biological behavior in individual tumors. To maximize the clinical benefit of these multiagent targeted therapies, it will be necessary to develop tools that allow clinicians to monitor tumor response during chemotherapy.

The goal of this study was to develop and validate a clinically applicable protocol that would allow monitoring of tumor response to targeted drug therapy by a cytology-based approach. The use of cytology has several inherent advantages. It is 
already widely accepted as a tool for the diagnosis of malignancy. Cytology specimens, specifically fine needle aspirations, can be obtained through minimally invasive procedures, even when accessing deep anatomic locations, without undue discomfort or risk to the patient. With this ability, clinicians can gauge tumor response to therapy over a period of time with successive samplings. Modifications to the patient's treatment regimen can then be made if the tumor becomes biochemically refractory to therapy. The use of a cytology-based test allows us to integrate morphology with immunohistochemistry, making it possible to factor out the effect of contaminating non-tumor cells such as stroma.

\section{Materials and methods}

\section{Cell Lines}

GIST 882 cell lines were maintained in T25 flasks in RPMI 1640 medium, supplemented with $10 \%$ fetal bovine serum. Activation of KIT and KIT-dependent proteins was evaluated by exposing confluent cells in serum-free media to DMSO (carrier negative control), imatinib $(1 \mu \mathrm{M})$, or RAD001 $(20 \mathrm{nM})$ for $48 \mathrm{~h}$. The GIST882 cells were then released from the T25 flasks by trypsinization and washed in phosphate-buffered saline (PBS) before preparation of cytology slides.

\section{Slide Preparation}

Harvested GIST cell lines were re-suspended in either PreservCyt fluid (Cytyc Corp., Marlborough, MA, USA) or saline. Thin-layer preparations were made using the ThinPrep 2000 (Cytyc, Marlborough, MA, USA) according to standard procedures. Cytocentrifuge slides (cytospins) were prepared using the Shandon Cytospin 3 (Thermo Electron Corp., Waltham, MA, USA) by centrifuging at 1330 r.p.m. for $6 \mathrm{~min}$.

\section{Immunocytochemistry}

Slides were fixed in $4 \%$ paraformaldehyde prepared in PBS for $10 \mathrm{~min}$. After rinsing in PBS, antigen retrieval was performed by microwaving the slides in citrate buffer (Zymed, South San Francisco, CA, USA) at $199^{\circ} \mathrm{F}$ for $10 \mathrm{~min}$, a further incubation at room temperature for $20 \mathrm{~min}$, followed by a rinse in PBS. Slides were pretreated with Peroxo-block (Invitrogen Corp., Carlsbad, CA, USA), washed with PBS-Tween $(0.05 \%)$, and blocked with CAS-block (Invitrogen Corp., Carlsbad, CA, USA). Primary antibodies were diluted in CAS-block and incubated as shown in Table 1. Secondary antibody incubation with a biotinylated pan-anti-mouse antibody was performed for $30 \mathrm{~min}$ at room temperature followed by incubation with a horseradish peroxidase-streptavidin enzyme conjugated complex for $30 \mathrm{~min}$ at room temperature. Detection was performed with an ABC reagent (Vector Laboratories, Burlingame, CA, USA), followed by a hematoxylin counterstain (1:1 dilution for $30 \mathrm{~s}$ ) and the placement of a coverslip.

\section{Microscopy}

All images were acquired using a Photometrics cooled CCD camera, with Biological Detection Systems image analysis software, from a Zeiss fluorescence microscope.

\section{Results}

Initial experiments were performed using cytospin preparations of the GIST 882 cell lines (Figure 1). Although several of the antibodies demonstrated strong reactivity to the GIST cells, background levels of reactivity, even in the absence of primary antibody incubation, remained high despite attempts to minimize non-specific reactivity by diluting the primary and secondary detection reagents.

An alternative method of slide preparation using thin-layer technology (ThinPrep) was then evaluated. This method utilizes proprietary reagents for cell fixation as well as proprietary equipment for thin-layer preparation. In contrast to cytospin-based slides, non-specific background reactivity was lower (Figure 2). Although robust staining was detectable with anti-KIT antibody, the phospho-antibodies, in general, showed much weaker levels of reactivity, and the signal strengths were not substantially improved when these antibodies were used at higher concentrations (data not shown). The one

Table 1 Primary antibodies

\begin{tabular}{llcr}
\hline Antibodies & Manufacturer & Dilution & Incubation \\
\cline { 3 - 4 } & & & Temperature ( ${ }^{\circ}$ C) \\
\hline C-KIT & DAKO (Carpinteria, CA, USA) & $1: 100$ & Room temperature \\
phospho-KIT & Zymed (South San Francisco, CA, USA) & 4 & $1 \mathrm{~h}$ \\
phospho-S6 (S235/236) & Cell Signaling Technology (Danvers, MA, USA) & $1: 50$ & Overnight \\
phospho-S6 (S240/244) & Cell Signaling Technology (Danvers, MA, USA) & $1: 50$ & Overnight \\
phospho-AKT & Cell Signaling Technology (Danvers, MA, USA) & $1: 50$ & 4 \\
Overnight & $1 \mathrm{~h}$ \\
\hline
\end{tabular}



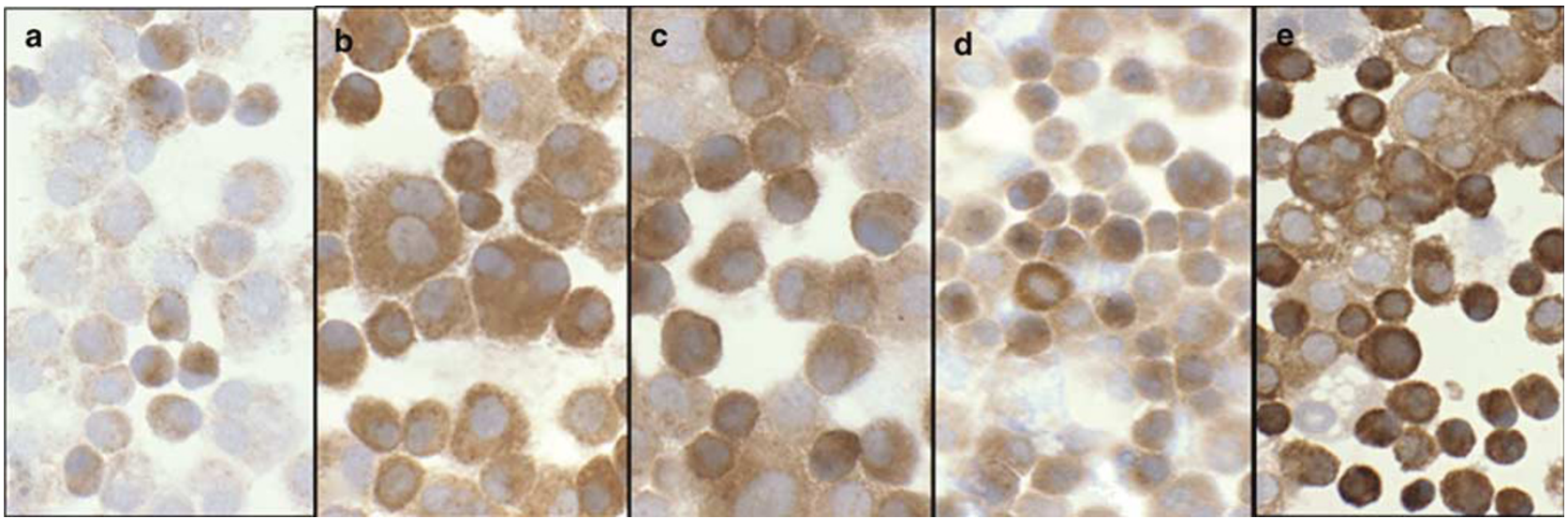

Figure 1 Cytospin preparations of cultured GIST882 cells without inhibitor. (a) Negative control (no primary antibody). Note the scattered non-specific staining. (b) Strong, diffuse immunoreactivity for KIT. (c) Strong, diffuse immunoreactivity for phospho-KIT. (d) Strong, focal immunoreactivity for phospho-AKT. (e) Strong, diffuse immunoreactivity for phospho-S6 (S240/244).

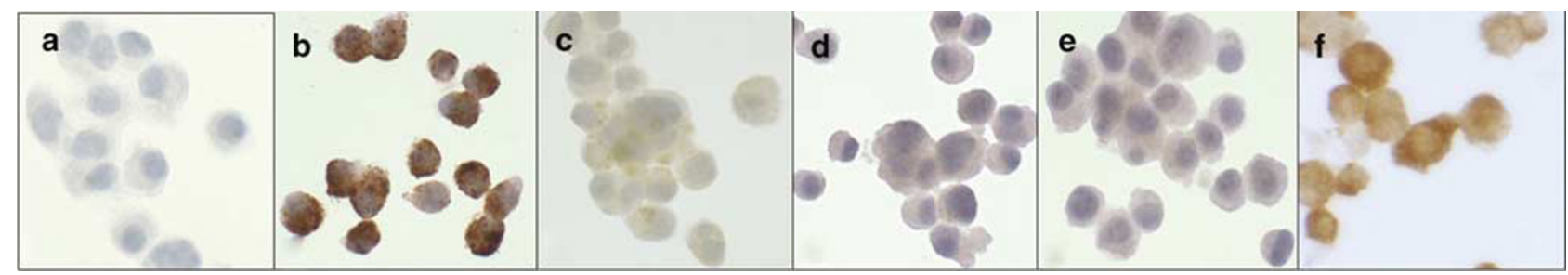

Figure 2 ThinPrep preparations of cultured GIST 822 cells without inhibitor. (a) Negative control (no antibody). Note the absence of nonspecific ('background') staining. (b) Strong, diffuse immunoreactivity for KIT. (c) Weak immunoreactivity for phospho-KIT. (d) Weak immunoreactivity for phospho-AKT. (e) Weak immunoreactivity for phospho-S6 (S240/244). (f) Strong, diffuse immunoreactivity for phospho-S6 (S235/236).

exception was the antibody directed against the phosphorylated serines at positions 235 and 236 of the S6 ribosomal protein. This particular antibody reproducibly demonstrated strong reactivity to the GIST 882 cells, which was substantially higher than background staining. As none of the other phosphoantibodies appeared to have strong signal intensities using the thin-layer technology, it was decided to focus on the phospho-S6 ribosomal protein for inhibitor studies.

In vitro inhibitor studies were performed to validate the specificity of the phospho-S6 (S235/ 236) antibody. GIST 882 cells were incubated for $48 \mathrm{~h}$ with DMSO (control) or with the RAD001 inhibitor of mTOR, which is upstream of S6. Control DMSO-treated GIST882 cells maintained strong reactivity with phospho-S6 (S235/236) (Figure 3). In contrast, RAD001-treated cells demonstrated a dramatic reduction in phospho-S6 antibody reactivity. Expression of total S6 protein was unaffected by RAD001 treatment (data not shown).

The utility of phospho-S6 as a readout for KIT kinase inhibition was then evaluated by treating GIST882 cells with imatinib (KIT kinase inhibitor) vs DMSO (carrier control) (Figure 4). DMSO treatment did not inhibit reactivity with phospho-S6 (S235/236), whereas imatinib treatment resulted in dramatic inhibition of phospho-S6 reactivity. However, imatinib treatment had no effect on total S6 expression (data not shown), indicating the selectivity of KIT inhibition for S6 biochemical inactivation.

\section{Discussion}

These studies represent initial steps towards validating a cytology-based immunocytochemical assay for monitoring of targeted therapy kinase response in GIST. The validations were performed by treating GIST882 cells with inhibitors of crucial signaling proteins involved in GIST cell proliferation and survival. Our findings show that clinically applicable, functional cytology assays can be used to monitor growth pathways that are important in GIST biology. The ability to measure the phosphorylation status of downstream effectors of the KIT oncoprotein, such as S6 ribosomal protein, will enable biological monitoring of clinical GIST response to targeted therapies such as imatinib and RAD001.

Clearly, functional assays of this nature are dependent on the availability of phopho-antibodies with high specificity and sensitivity for their target epitopes. Although many such antibodies have been 


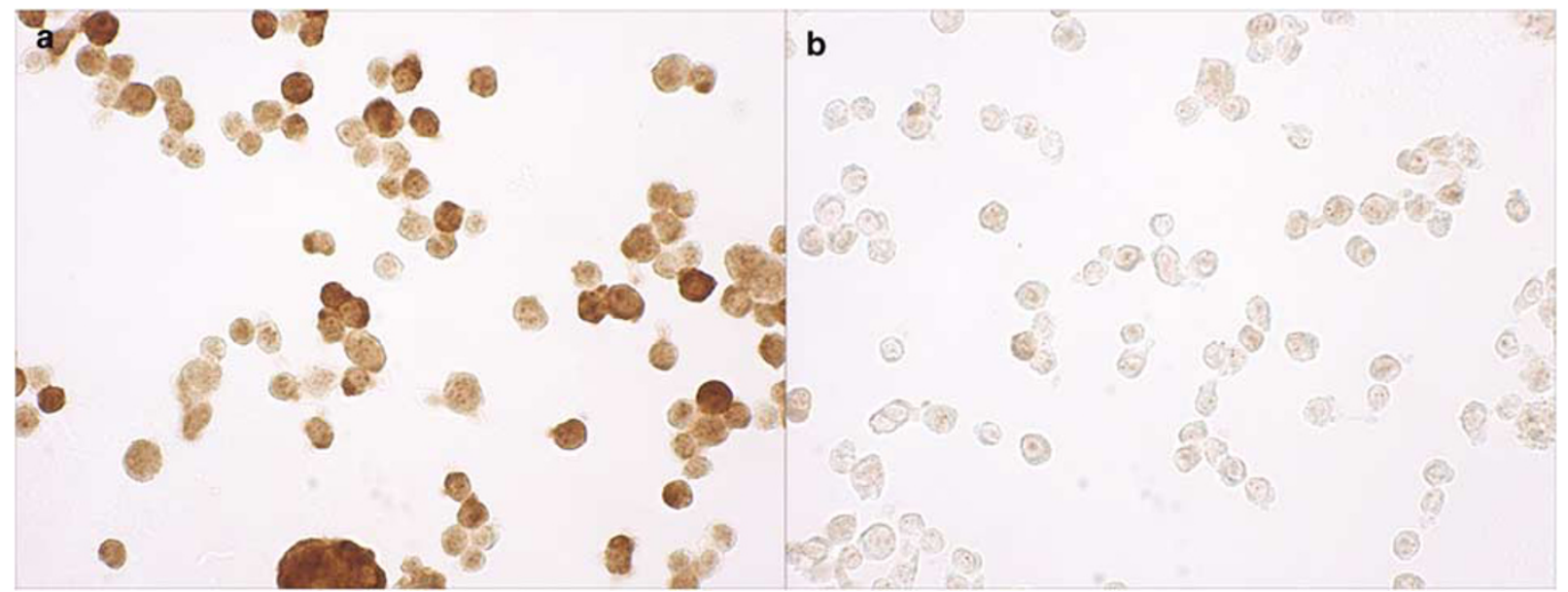

Figure 3 ThinPrep preparations of cultured GIST882 cells with and without mTOR inhibitor (RAD001). (a) Strong immunoreactivity for phospho-S6 (S235/236) after control treatment with DMSO. (b) Immunoreactivity for phospho-S6 (S235/236) is virtually abolished after treatment with upstream inhibitor RAD001.

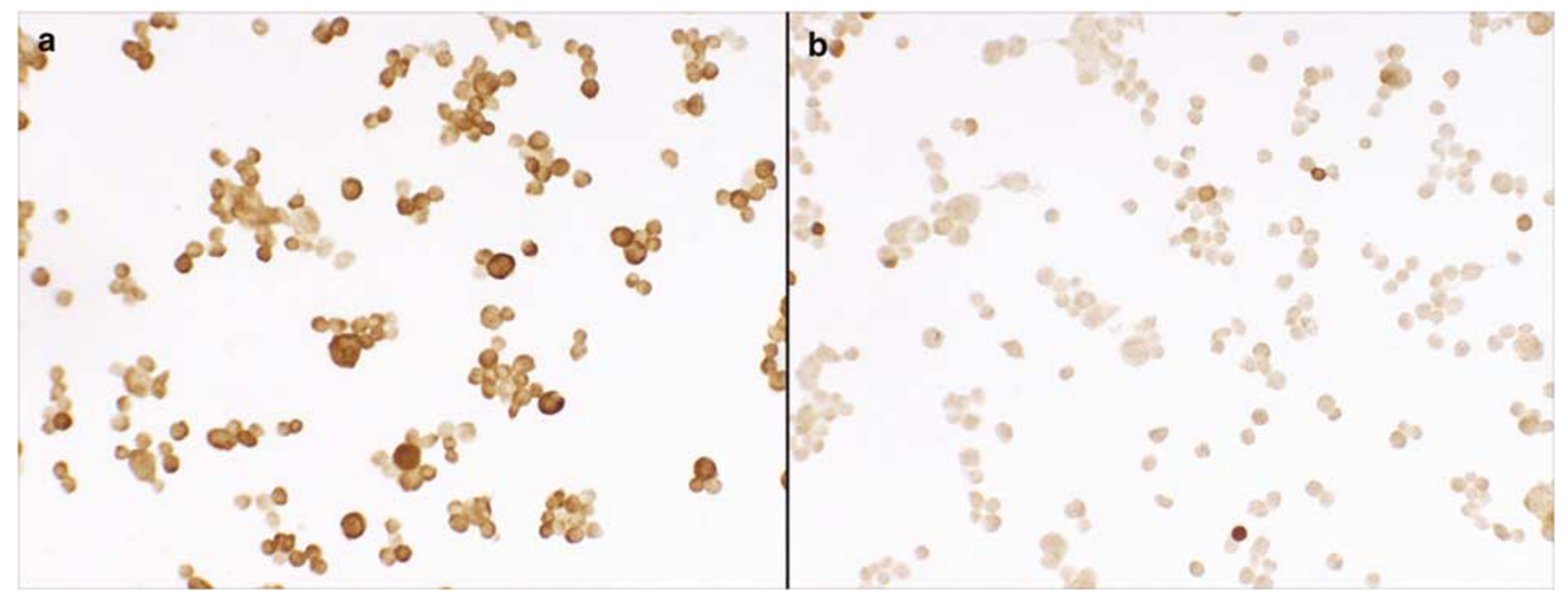

Figure 4 ThinPrep preparations of cultured GIST882 cells with and without KIT kinase inhibitor (imatinib). (a) Strong immunoreactivity for phospho-S6 (235/236) after control treatment with DMSO. (b) Immunoreactivity for phospho-S6 (235/236) is substantially reduced after treatment with KIT kinase inhibitor, although scattered cells continue to express phospho-S6.

used successfully in immunoblotting studies, it has been challenging to create antibodies specific for phosphorylated-tyrosine, serine or threonine epitopes-which do not crossreact with closely related phospho-epitopes in family members of the intended target protein-and which therefore have sufficient specificity for routine immunohistochemical applications. Of the four phospho-protein antibodies assayed in this study, only the reagent for phospho-S6 showed strong and highly specific reactivity.

More work remains to be done to optimize conditions and generate new antibodies so as to extend these assays to other crucial signaling pathways in oncology. The general approach described here, however, can be extended to any other signaling pathway that is dependent on a given drug target in human cancer cells. In addition, these methods can be applied to monitor drug response in mouse xenografts or transgenic models, for example, using nude mice injected with GIST cell lines. Once these preclinical methodologies have been more fully validated, we anticipate the use of similar strategies in monitoring patient response to targeted kinase inhibitor therapies in clinical trials, andultimately-in routine testing to determine the most suitable kinase inhibitor for a given patient.

The ability to measure biochemical responsiveness (or lack of responsiveness) of tumors to targeted chemotherapy will be an extremely useful tool for oncologists. Currently, tumors are not routinely sampled during therapy, and responsiveness to therapy is monitored by clinical or radiological means. The ability to measure biochemical response at crucial time points during therapy would give clinicians the ability to detect resistance earlier and 
allow rational modification or augmentation of therapy for an improved clinical response.

\section{Acknowledgement}

This work was supported in part by a Cytopathology Research Seed Award from the American Society of Cytopathology Foundation and by a grant from Cytyc Corp. (Marlborough, MA, USA).

\section{References}

1 Eisenberg BL. Imatinib mesylate: a molecularly targeted therapy for gastrointestinal stromal tumors. Oncology 2003;17:1615-1620.

2 Duensing A, Medeiros F, McConarty B, et al. Mechanisms of oncogenic KIT signal transduction in primary gastrointestinal stromal tumors (GISTs). Oncogene 2004;23:3999-4006. 